\title{
The spectrally bounded linear maps on operator algebras
}

\author{
by \\ JiAnLian Cui (Beijing, Taiyuan and Linfen) \\ and JinchuAN Hou (Linfen and Taiyuan)
}

\begin{abstract}
We show that every spectrally bounded linear map $\Phi$ from a Banach algebra onto a standard operator algebra acting on a complex Banach space is squarezero preserving. This result is used to show that if $\Phi_{2}$ is spectrally bounded, then $\Phi$ is a homomorphism multiplied by a nonzero complex number. As another application to the Hilbert space case, a classification theorem is obtained which states that every spectrally bounded linear bijection $\Phi$ from $\mathcal{B}(H)$ onto $\mathcal{B}(K)$, where $H$ and $K$ are infinite-dimensional complex Hilbert spaces, is either an isomorphism or an anti-isomorphism multiplied by a nonzero complex number. If $\Phi$ is not injective, then $\Phi$ vanishes at all compact operators.
\end{abstract}

1. Introduction. Over the past decades, there has been a considerable interest in the study of linear maps on operator algebras that preserve certain properties of operators. In particular, a problem how to characterize linear maps that preserve the spectrum of each operator has attracted the attention of many mathematicians. In [11], Jafarian and Sourour proved that a surjective linear map preserving spectrum from $\mathcal{B}(X)$ onto $\mathcal{B}(Y)$, where $X$ and $Y$ are Banach spaces, is either an isomorphism or an anti-isomorphism. Aupetit and Mouton [3] extended this result to primitive Banach algebras with minimal ideals. It is shown in [13] that every point spectrum preserving and surjective linear map on $\mathcal{B}(X)$ is an automorphism. Brešar and Šemrl [5] proved that a linear surjective map preserving spectral radius on $\mathcal{B}(X)$ is either an automorphism or an anti-automorphism multiplied by a scalar with modulus 1 . For some other papers concerning this type of linear preservers, see $[1,2,4,6-8,10-11,13,16-18]$.

A natural and interesting question is to ask how to classify the surjective linear maps $\Phi$ on $\mathcal{B}(X)$ which are spectrally bounded, i.e., there exists a positive constant $M$ such that $r(\Phi(T)) \leq M r(T)$ for every $T \in \mathcal{B}(X)$, where $r(T)$ denotes the spectral radius of $T$. In the case that $M=1$ we say that $\Phi$

2000 Mathematics Subject Classification: Primary 47B48, 47L10, 47A10.

Key words and phrases: spectral radius, Jordan homomorphism, isomorphism, Banach algebras.

This work is supported by NNSFC and PNSFS. 
is spectral radius nonincreasing. Note that the study of spectrally bounded linear maps can be reduced to that of the spectral radius nonincreasing ones. Indeed, let $\Psi=(1 / M) \Phi$; then $r(\Psi(T))=(1 / M) r(\Phi(T)) \leq r(T)$, that is, $\Psi$ is spectral radius nonincreasing.

A related reference is [14], where Šmrl proved that a unital bijective linear map on $\mathcal{B}(H)$ with $H$ infinite-dimensional is spectrally bounded if and only if it is either an automorphism or an anti-automorphism. One of our main purposes in this note is to improve Šemrl's result above by omitting the assumption that $\Phi$ is unital.

When the Hilbert space $H$ is finite-dimensional, together with the discussion of Šemrl [14, Remark 4], one can easily check that a bijective linear map $\Phi$ on $\mathcal{B}(H)$ is spectrally bounded if and only if $\Phi$ has the form $\Phi(T)=c \varphi(T)+(\Phi(I)-c I) \operatorname{tr} T / n$ for every $T \in \mathcal{B}(H)$, where $\varphi$ is either an automorphism or an anti-automorphism of $\mathcal{B}(H), c$ is a nonzero complex number and $n=\operatorname{dim} H$. So when we discuss the spectrally bounded linear maps, we may always assume that the Hilbert space $H$ is infinitedimensional.

Now we describe the main results of this note. In Section 2, we prove in a quite general framework that a spectrally bounded linear map from a unital complex Banach algebra onto a standard operator algebra acting on a complex Banach space preserves square-zero elements (see Theorem 2.1), which generalizes the first step of the proof of Šemrl's result in [14] mentioned above. This allows us to prove that a two-fold spectrally bounded surjective linear map from a unital complex Banach algebra onto a standard operator algebra acting on a complex Banach space is a homomorphism multiplied by a nonzero complex number (Theorem 2.2). Here a linear map $\Phi: \mathcal{A} \rightarrow \mathcal{B}$ is said to be two-fold spectrally bounded if $\Phi_{2}: \mathcal{A} \otimes M_{2}(\mathbb{C}) \rightarrow$ $\mathcal{B} \otimes M_{2}(\mathbb{C})$ defined by $\Phi_{2}\left(\left(T_{i j}\right)\right)=\left(\Phi\left(T_{i j}\right)\right)$ is spectrally bounded. Section 3 concerns applications of Theorem 2.1 to the Hilbert space case. We show that a linear bijection $\Phi$ from $\mathcal{B}(H)$ onto $\mathcal{B}(K)$, where $H$ and $K$ are infinitedimensional complex Hilbert spaces, is spectrally bounded if and only if there exist a nonzero complex number $d$ and an invertible operator $A \in$ $\mathcal{B}(H, K)$ such that either $\Phi(T)=d A T A^{-1}$ for all $T \in \mathcal{B}(H)$ or $\Phi(T)=$ $d A T^{\operatorname{tr}} A^{-1}$ for all $T \in \mathcal{B}(H)$ (Theorem 3.1). If the injectivity assumption on $\Phi$ is omitted, then either $\Phi$ has one of the above forms or $\Phi$ vanishes at every compact operator (Theorem 3.3). In particular, if $H$ is separable, then $\Phi$ is surjective and spectrally bounded if and only if it is either an isomorphism or an anti-isomorphism multiplied by a nonzero scalar. From our arguments, we also answer affirmatively a question raised by Šemrl in [15] where he gave a characterization of unital bijections on $\mathcal{B}(H)$ which preserve squarezero operators and asked whether or not the "unital" assumption can be omitted. 
Let us fix some notations. Let $\mathcal{B}(X)$ and $\mathcal{F}(X)$ be the sets of all bounded linear operators and of all finite rank bounded linear operators on the Banach space $X$, respectively. A subalgebra $\mathcal{B}$ in $\mathcal{B}(X)$ is called a standard operator algebra if $\mathcal{B}$ is closed and contains the identity operator and $\mathcal{F}(X)$. For $T \in \mathcal{B}(X)$, we denote by $\mathcal{R}(T)$ and $\operatorname{ker}(T)$ the range and kernel of $T$, respectively. If $T^{2}=T$, we say $T$ is an idempotent operator. Throughout this paper, we denote by $x \otimes f$ the bounded linear operator on $X$ defined for any $x \in X$ and $f \in X^{*}$ by $(x \otimes f)(z)=\langle z, f\rangle x$ for every $z \in X$, where $\langle z, f\rangle$ is the value of $f$ at $z$. Note that this operator is of rank one whenever both $x$ and $f$ are nonzero, and that every operator of rank one can be written in this form. By a projection we mean a self-adjoint idempotent in $\mathcal{B}(H)$, where $H$ is a Hilbert space.

2. General results. In this section we consider the general case of spectrally bounded linear maps from a Banach algebra onto a standard operator algebra on a complex Banach space. The following is our main result.

Theorem 2.1. Let $\mathcal{A}$ be a unital complex Banach algebra and $\mathcal{B}$ be a standard operator algebra on a complex Banach space $X$. Assume that $\Phi: \mathcal{A} \rightarrow \mathcal{B}$ is a surjective linear map. If $\Phi$ is spectrally bounded, then $\Phi$ preserves square-zero elements.

Proof. By the discussion in the introduction, we may assume that $\Phi$ is spectral radius nonincreasing. We divide the proof into two steps. We mention that the idea of the proof of Step 1 is the same as an idea used in $[5]$.

STEP 1. Let $A \in \mathcal{A}$ be such that $A^{k}=0$ for some $k \geq 2$. If $B \in \mathcal{B}$ satisfies $B Q^{i} B=0, i=0,1, \ldots, k-1$, where $Q=\Phi(A)$, then

$$
r\left(\lambda Q^{k}+B Q^{k-1}+Q B Q^{k-2}+\ldots+Q^{k-1} B\right)=0
$$

for every complex number $\lambda$.

Let $B_{1}=B Q^{k-1}+Q B Q^{k-2}+\ldots+Q^{k-1} B$ and $B_{2}=Q^{k}$. Since $r(B+\lambda Q)^{k}$ $=r\left[(B+\lambda Q)^{k}\right]$ and $B^{2}=B Q B=B Q^{2} B=\ldots=B Q^{k-2} B=0$, it follows that

$$
r(B+\lambda Q)^{k}=|\lambda|^{k-1} r\left(B_{1}+\lambda B_{2}\right) .
$$

As $\Phi$ is surjective, there exists $C \in \mathcal{A}$ such that $\Phi(C)=B$. Moreover, since $\Phi$ is spectral radius nonincreasing, we have

$$
r(B+\lambda Q)^{k}=r(\Phi(C+\lambda A))^{k} \leq r(C+\lambda A)^{k} .
$$

As $A^{k}=0$, it follows that

$$
r(C+\lambda A)^{k}=r\left[(C+\lambda A)^{k}\right]=r\left(C_{0}+\lambda C_{1}+\ldots+\lambda^{k-1} C_{k-1}\right),
$$


where $C_{0}=C^{k}, C_{1}=C^{k-1} A+\ldots+A C^{k-1}, \ldots, C_{k-1}=A^{k-1} C+\ldots+$ $C A^{k-1}$. Thus we have shown that

$$
|\lambda|^{k-1} r\left(B_{1}+\lambda B_{2}\right) \leq r\left(C_{0}+\lambda C_{1}+\ldots+\lambda^{k-1} C_{k-1}\right) .
$$

Therefore, for any complex $\lambda$ satisfying $|\lambda| \geq 1$, we have

$$
\begin{aligned}
r\left(B_{1}+\lambda B_{2}\right) & \leq|\lambda|^{-k+1} r\left(C_{0}+\lambda C_{1}+\ldots+\lambda^{k-1} C_{k-1}\right) \\
& \leq\left\|C_{0}\right\|+\left\|C_{1}\right\|+\ldots+\left\|C_{k-1}\right\| .
\end{aligned}
$$

On the other hand, for every complex $\lambda$ satisfying $|\lambda| \leq 1$, one gets

$$
r\left(B_{1}+\lambda B_{2}\right) \leq\left\|B_{1}+\lambda B_{2}\right\| \leq\left\|B_{1}\right\|+\left\|B_{2}\right\| .
$$

Thus the function $\lambda \mapsto r\left(B_{1}+\lambda B_{2}\right)$ is bounded on $\mathbb{C}$. As it is subharmonic, the Liouville theorem for subharmonic functions [3] shows that $r\left(B_{1}+\lambda B_{2}\right)$ $=r\left(B_{1}\right)$ for every complex $\lambda$. Observing that $B_{1} B=0$ it is easy to see that $B_{1}^{2}=D_{k-2} B Q^{k-2}+D_{k-3} B Q^{k-3}+\ldots+D_{0} B$ for some $D_{i} \in \mathcal{B}$ and so $B_{1}^{2} Q B=B_{1}^{2} B=0$. This further implies that $B_{1}^{3}$ has the form $B_{1}^{3}=$ $E_{k-3} B Q^{k-3}+E_{k-2} B Q^{k-2}+\ldots+E_{0} B$ for some $E_{i} \in \mathcal{B}$, and consequently, $B_{1}^{3} Q^{2} B=B_{1}^{3} Q B=B_{1}^{3} B=0$. Repeating this procedure one shows that $B_{1}^{k+1}=0$. So $r\left(B_{1}+\lambda B_{2}\right)=0$ for every complex $\lambda$, as desired.

Step 2. If $A \in \mathcal{A}$ and $A^{2}=0$, then $\Phi(A)^{2}=0$.

Based on Step 1, an argument similar to that in Step 5 of the proof of the main results in [5], where $\mathcal{B}(X)$ is replaced by $\mathcal{A}$ or $\mathcal{B}$, shows that, for every $k \geq 2$ and every $A \in \mathcal{A}$ with $A^{k}=0$, we have $\Phi(A)^{2 k-1}=0$. So for every $A \in \mathcal{A}$ satisfying $A^{2}=0$, we have $\Phi(A)^{3}=0$. Assume that there exists some $A \in \mathcal{A}$ with $A^{2}=0$ but $\Phi(A)^{2} \neq 0$. Let $\Phi(A)=Q$. It follows that $p(Q) \neq 0$ for every complex polynomial $p$ of degree not exceeding 2 . Kaplansky's theorem on local algebraic operators tells us that there is $u \in X$ such that the vectors $u, Q u$ and $Q^{2} u$ are linearly independent. Therefore, $u \notin M=\operatorname{span}\left\{Q u, Q^{2} u-u\right\}$. Hence there exists a linear functional $f \in X^{*}$ such that $f(u)=f\left(Q^{2} u\right)=1$ and $f(Q u)=0$. Let $B=\left(Q^{2} u-u\right) \otimes f$. Then a straightforward computation shows that $B^{2}=B Q B=0$. So, by Step 1 , we have $r\left(Q^{2}+B Q+Q B\right)=0$. On the other hand, one can easily check

$$
\left(Q^{2}+B Q+Q B\right)(u-Q u)=u-Q u,
$$

so $r\left(Q^{2}+B Q+Q B\right) \geq 1$, which is a contradiction. Hence $\Phi$ preserves square-zero elements.

Applying Theorem 2.1, we can prove the following results.

TheOREm 2.2. Let $\mathcal{A}$ be a unital complex Banach algebra and $\mathcal{B}$ be a standard operator algebra on a complex Banach space. Assume that $\Phi$ : $\mathcal{A} \rightarrow \mathcal{B}$ is a surjective linear map. Then $\Phi_{2}: \mathcal{A} \otimes M_{2}(\mathbb{C}) \rightarrow \mathcal{B} \otimes M_{2}(\mathbb{C})$ is spectrally bounded if and only if $\Phi$ is a homomorphism multiplied by a nonzero complex number. 
Proof. The sufficiency is clear. Now let us check the necessity. Assume that $\Phi_{2}: \mathcal{A} \otimes M_{2}(\mathbb{C}) \rightarrow \mathcal{B} \otimes M_{2}(\mathbb{C})$ is spectrally bounded. By Theorem 2.1, $\Phi_{2}$ preserves square-zero elements. Let $C \in \mathcal{A}$ be invertible. For any $A \in \mathcal{A}$, since

$$
\left(\begin{array}{cc}
A & C \\
-C^{-1} A^{2} & -C^{-1} A C
\end{array}\right)^{2}=0
$$

we have

$$
\left(\begin{array}{cc}
\Phi(A) & \Phi(C) \\
-\Phi\left(C^{-1} A^{2}\right) & -\Phi\left(C^{-1} A C\right)
\end{array}\right)^{2}=0 .
$$

So

$$
\Phi(A)^{2}-\Phi(C) \Phi\left(C^{-1} A^{2}\right)=0
$$

and

$$
\Phi(A) \Phi(C)-\Phi(C) \Phi\left(C^{-1} A C\right)=0 .
$$

Letting $C=I$ in $(2.2)$ gives $\Phi(I) \Phi(A)=\Phi(A) \Phi(I)$. Since $\Phi$ is surjective and also spectral radius nonincreasing, and since $\mathcal{B}$ is a standard operator algebra, we must have $\Phi(I)=c I$ for some complex number $c$ with $|c| \leq 1$. We claim $c \neq 0$. Otherwise, taking $C=I$ in $(2.1)$ gives $\Phi(A)^{2}=0$ for every $A \in \mathcal{A}$, which contradicts the surjectivity of $\Phi$. So, without loss of generality, we may assume that $\Phi(I)=I$. Now it is clear that $\Phi\left(A^{2}\right)=\Phi(A)^{2}$, that is, $\Phi$ is a Jordan homomorphism. By taking $A=I$ in (2.1), we see that $\Phi$ also preserves invertibility. So (2.2) implies

$$
\Phi\left(C^{-1} A C\right)=\Phi(C)^{-1} \Phi(A) \Phi(C) .
$$

Since $\Phi$ is Jordan, we have $\Phi(C A C)=\Phi(C) \Phi(A) \Phi(C)$ for any $A, C \in \mathcal{A}$. Hence

$$
\Phi\left(A C^{2}\right)=\Phi\left(C^{-1} C A C C\right)=\Phi(C)^{-1} \Phi(C A C) \Phi(C)=\Phi(A) \Phi(C)^{2} .
$$

Next, choosing any nonzero $\lambda \in \mathbb{C}$ so that $\lambda-C$ is invertible, we get, replacing $C$ by $\lambda-C$ in $(2.3)$,

$$
\Phi(A C)=\Phi(A) \Phi(C)
$$

for all $A$ and invertible $C$ in $\mathcal{A}$. When $C$ is not invertible, take $\lambda \in \mathbb{C}$ so that $\lambda-C$ is invertible; then $\Phi(A(\lambda-C))=\Phi(A)(\lambda-\Phi(C))$, which again implies that $\Phi(A C)=\Phi(A) \Phi(C)$. Therefore, $\Phi$ is a homomorphism from $\mathcal{A}$ onto $\mathcal{B}$.

Corollary 2.3. Let $\mathcal{A}$ be a unital complex Banach algebra and $\mathcal{B}$ be a standard operator algebra on a complex Banach space. Assume that $\Phi: \mathcal{A} \rightarrow \mathcal{B}$ is a bijective linear map. Then $\Phi_{2}: \mathcal{A} \otimes M_{2}(\mathbb{C}) \rightarrow \mathcal{B} \otimes M_{2}(\mathbb{C})$ is spectrally bounded if and only if $\Phi$ is an isomorphism multiplied by a nonzero complex number. 
If $\mathcal{A}$ is a standard operator algebra, then $\Phi$ has a more concrete characterization.

Corollary 2.4. Let $\mathcal{A}$ and $\mathcal{B}$ be two standard operator algebras on a complex Banach space $X$. Assume that $\Phi: \mathcal{A} \rightarrow \mathcal{B}$ is a bijective linear map. Then $\Phi_{2}: \mathcal{A} \otimes M_{2}(\mathbb{C}) \rightarrow \mathcal{B} \otimes M_{2}(\mathbb{C})$ is spectrally bounded if and only if there exists a complex number $c$ and an invertible operator $A \in \mathcal{B}(X)$ such that $\Phi(T)=c A T A^{-1}$ for every operator $T \in \mathcal{A}$.

Proof. It is clear that we need only check the necessity. Assume that $\Phi_{2}$ is spectrally bounded. By Theorem $2.2, \Phi$ is a scalar multiple of an isomorphism. Since every isomorphism between standard operator algebras is spatial, there exists an invertible operator $A \in \mathcal{B}(X)$ such that $\Phi(T)=$ $c A T A^{-1}$ for every $T \in \mathcal{A}$.

3. Application to Hilbert space case. Let $H$ and $K$ be two infinitedimensional complex Hilbert spaces. Applying the results of Section 2, we can get a complete classification of the spectrally bounded linear maps from $\mathcal{B}(H)$ onto $\mathcal{B}(K)$ without the assumption that $\Phi(I)=I$. The following theorem is our main result in this section.

Theorem 3.1. Let $H$ and $K$ be two infinite-dimensional Hilbert spaces. Assume that $\Phi: \mathcal{B}(H) \rightarrow \mathcal{B}(K)$ is a bijective linear map. Then the following conditions are equivalent.

(1) $\Phi$ is spectrally bounded.

(2) There exists a nonzero complex number $d$ and a bounded linear map $\Psi: \mathcal{B}(H) \rightarrow \mathcal{B}(K)$ preserving idempotents such that $\Phi=d \Psi$.

(3) $\Phi$ is a Jordan isomorphism multiplied by a nonzero complex number.

(4) $\Phi$ is either an isomorphism or an anti-isomorphism multiplied by a nonzero complex number.

(5) There exists a nonzero complex number $d$ and an invertible operator $A \in \mathcal{B}(H, K)$ such that either $\Phi(T)=d A T A^{-1}$ for all $T \in \mathcal{B}(H)$ or $\Phi(T)=$ $d A T^{\mathrm{tr}} A^{-1}$ for all $T \in \mathcal{B}(H)$, where $T^{\mathrm{tr}}$ denotes the transpose of $T$ relative to a fixed but arbitrary orthonormal basis of $H$.

To prove this theorem, the following lemma is needed.

Lemma 3.2. Suppose that $\Phi: \mathcal{B}(H) \rightarrow \mathcal{B}(K)$ is a surjective linear map which preserves square-zero operators. Then

$$
\Phi(R)^{2} \Phi(I)=\Phi(I) \Phi(R)^{2}
$$

for all idempotents $R \in \mathcal{B}(H)$.

Proof. Let $H$ be a direct sum of two closed infinite-dimensional linear subspaces $H_{1}$ and $H_{2}$ (note that we do not assume $H_{1}$ and $H_{2}$ are orthogonal). Let $P$ and $Q=I-P$ be the idempotents corresponding to this direct 
sum decomposition, that is, $\mathcal{R}(P)=H_{1}$ and $\operatorname{ker}(P)=H_{2}$. Assume that operators $A, B \in B(H)$ satisfy $P A P=A$ and $Q B Q=B$. It follows from [12, Theorem 2] that $A$ and $B$ can be written as sums of five operators with square zero. Say $A=A_{1}+A_{2}+A_{3}+A_{4}+A_{5}$ and $B=B_{1}+B_{2}+B_{3}+B_{4}+B_{5}$, with $P A_{i} P=A_{i}$ and $Q B_{i} Q=B_{i}(i=1, \ldots, 5)$. Clearly, $\left(A_{i}+B_{j}\right)^{2}=0$. Consequently, we have $\Phi\left(A_{i}\right) \Phi\left(B_{j}\right)+\Phi\left(B_{j}\right) \Phi\left(A_{i}\right)=0$, which further yields

$$
\Phi(A) \Phi(B)+\Phi(B) \Phi(A)=0 .
$$

In other words, we have

$$
\Phi(P A P) \Phi((I-P) B(I-P))+\Phi((I-P) B(I-P)) \Phi(P A P)=0
$$

for every $A, B \in \mathcal{B}(H)$.

We claim that

$$
\Phi(R) \Phi(I)+\Phi(I) \Phi(R)=2 \Phi(R)^{2} \quad \text { for all idempotents } R .
$$

If $R \in \mathcal{B}(H)$ is an idempotent such that both its range and kernel are infinite-dimensional, then by (3.2) with $A=B=I$ we get

$$
\Phi(R) \Phi(I-R)+\Phi(I-R) \Phi(R)=0,
$$

which implies (3.3) immediately.

If $R$ has a finite-dimensional image, take an idempotent $P_{1}$ with both range and kernel infinite-dimensional such that $P_{1} \perp R$. Then

$$
\begin{aligned}
& \Phi\left(P_{1}\right) \Phi(R)+\Phi(R) \Phi\left(P_{1}\right) \\
& =\Phi\left(P_{1} P_{1} P_{1}\right) \Phi\left(\left(I-P_{1}\right) R\left(I-P_{1}\right)\right)+\Phi\left(\left(I-P_{1}\right) R\left(I-P_{1}\right)\right) \Phi\left(P_{1} P_{1} P_{1}\right)=0 .
\end{aligned}
$$

Therefore,

$$
\Phi\left(P_{1}+R\right)^{2}=\Phi\left(P_{1}\right)^{2}+\Phi(R)^{2}
$$

and

$$
\begin{aligned}
\Phi(R) \Phi(I) & +\Phi(I) \Phi(R) \\
= & \Phi\left(P_{1}+R-P_{1}\right) \Phi(I)+\Phi(I) \Phi\left(P_{1}+R-P_{1}\right) \\
= & \Phi\left(P_{1}+R\right) \Phi(I)+\Phi(I) \Phi\left(P_{1}+R\right)-\Phi\left(P_{1}\right) \Phi(I)-\Phi(I) \Phi\left(P_{1}\right) \\
= & 2 \Phi\left(P_{1}+R\right)^{2}-2 \Phi\left(P_{1}\right)^{2}=2 \Phi(R)^{2} .
\end{aligned}
$$

If $R$ has finite-dimensional kernel, then

$$
\Phi(I-R) \Phi(I)+\Phi(I) \Phi(I-R)=2 \Phi(I-R)^{2},
$$

and hence

$$
\Phi(R) \Phi(I)+\Phi(I) \Phi(R)=2 \Phi(R)^{2}
$$

completing the proof of (3.3). Thus, for any idempotent $R \in \mathcal{B}(H)$, we have

$$
\Phi(R)^{2} \Phi(I)+\Phi(R) \Phi(I) \Phi(R)=2 \Phi(R)^{3}
$$

and 


$$
\Phi(I) \Phi(R)^{2}+\Phi(R) \Phi(I) \Phi(R)=2 \Phi(R)^{3}
$$

which implies that $\Phi(R)^{2} \Phi(I)=\Phi(I) \Phi(R)^{2}$.

Theorem 3.3. Let $H$ and $K$ be two infinite-dimensional Hilbert spaces. Assume that $\Phi: \mathcal{B}(H) \rightarrow \mathcal{B}(K)$ is a surjective linear map. If $\Phi$ is spectrally bounded, then either $\Phi(T)=0$ for all compact operators $T \in \mathcal{B}(H)$ or $\Phi$ is injective. In the last case, there exists a nonzero complex number $d$ and an invertible operator $A \in \mathcal{B}(H, K)$ such that either $\Phi(T)=d A T A^{-1}$ for all $T \in \mathcal{B}(H)$ or $\Phi(T)=d A T^{\operatorname{tr}} A^{-1}$ for all $T \in \mathcal{B}(H)$, where $T^{\mathrm{tr}}$ denotes the transpose of $T$ relative to a fixed but arbitrary orthonormal basis of $H$.

Proof. Assume that $\Phi$ is spectrally bounded. By Theorem 2.1, $\Phi$ preserves square-zero operators. We may assume that $\Phi$ is spectral radius nonincreasing by the discussion in the introduction.

Claim 1. For any orthogonal idempotents $P_{1}$ and $P_{2}$ in $\mathcal{B}(H)$, we have

$$
\Phi\left(P_{1}+P_{2}\right)^{2}=\Phi\left(P_{1}\right)^{2}+\Phi\left(P_{2}\right)^{2} .
$$

It is easily seen from the proof of Lemma 3.2 that (3.4) is true if $\operatorname{dim} \mathcal{R}\left(P_{i}\right)$ $=\operatorname{dim} \mathcal{R}\left(I-P_{i}\right)=\infty$ for $i=1$ or 2 . There are four cases left to check:

(i) $\operatorname{dim} \mathcal{R}\left(P_{1}\right)<\infty, \operatorname{dim} \mathcal{R}\left(P_{2}\right)<\infty$;

(ii) $\operatorname{dim} \mathcal{R}\left(P_{1}\right)<\infty, \operatorname{dim} \mathcal{R}\left(I-P_{2}\right)<\infty$;

(iii) $\operatorname{dim} \mathcal{R}\left(I-P_{1}\right)<\infty, \operatorname{dim} \mathcal{R}\left(P_{2}\right)<\infty$;

(iv) $\operatorname{dim} \mathcal{R}\left(I-P_{1}\right)<\infty, \operatorname{dim} \mathcal{R}\left(I-P_{2}\right)<\infty$.

In case (i), we can find an idempotent $P_{3}$ orthogonal to $P_{1}+P_{2}$ with $\operatorname{dim} \mathcal{R}\left(P_{3}\right)=\operatorname{dim} \mathcal{R}\left(I-P_{3}\right)=\infty$. Thus

$$
\begin{aligned}
\Phi\left(P_{1}+P_{2}\right)^{2}+\Phi\left(P_{3}\right)^{2} & =\Phi\left(P_{1}+P_{2}+P_{3}\right)^{2}=\Phi\left(P_{1}\right)^{2}+\Phi\left(P_{2}+P_{3}\right)^{2} \\
& =\Phi\left(P_{1}\right)^{2}+\Phi\left(P_{2}\right)^{2}+\Phi\left(P_{3}\right)^{2},
\end{aligned}
$$

so $\Phi\left(P_{1}+P_{2}\right)^{2}=\Phi\left(P_{1}\right)^{2}+\Phi\left(P_{2}\right)^{2}$. The remaining cases are similar.

Claim 2. $\Phi(I)=c I$ for some nonzero complex number $c$.

We first prove that $\Phi$ is bounded. Since $\Phi$ is a spectral radius nonincreasing surjection and $\mathcal{B}(K)$ is semisimple, it follows from Aupetit [2] that $\Phi$ is bounded.

Let $C \in \mathcal{B}(H)$ be a linear combination of orthogonal projections, that is, $C=\sum_{i=1}^{n} \alpha_{i} P_{i}$, where $\left\{P_{i}\right\}_{i=1}^{n}$ is an orthogonal set of projections. Then $\Phi(C)^{2}=\sum_{i=1}^{n} \alpha_{i}^{2} \Phi\left(P_{i}\right)^{2}$ by (3.4). It follows that $\Phi(C)^{2} \Phi(I)=\Phi(I) \Phi(C)^{2}$ by Lemma 3.2. Now suppose $D \in \mathcal{B}(H)$ is self-adjoint; then $D$ is a limit of linear combinations of orthogonal projections. Since $\Phi$ is bounded and linear, we have $\Phi(D)^{2} \Phi(I)=\Phi(I) \Phi(D)^{2}$. Let $C, D \in \mathcal{B}(H)$ be self-adjoint. Then $\Phi(C+D)^{2} \Phi(I)=\Phi(I) \Phi(C+D)^{2}$, which yields

$$
(\Phi(C) \Phi(D)+\Phi(D) \Phi(C)) \Phi(I)=\Phi(I)(\Phi(C) \Phi(D)+\Phi(D) \Phi(C)) .
$$


For any $T \in \mathcal{B}(H)$, there exist self-adjoint operators $C$ and $D$ such that $T=C+i D$. Because

$$
\begin{aligned}
& \Phi(C+i D)^{2} \Phi(I) \\
& =\Phi(C)^{2} \Phi(I)-\Phi(D)^{2} \Phi(I)+i(\Phi(C) \Phi(D)+\Phi(D) \Phi(C)) \Phi(I) \\
& =\Phi(I) \Phi(C+i D)^{2}
\end{aligned}
$$

we see that

$$
\Phi(T)^{2} \Phi(I)=\Phi(I) \Phi(T)^{2}
$$

for every $T \in \mathcal{B}(H)$, and consequently $S^{2} \Phi(I)=\Phi(I) S^{2}$ for all $S \in \mathcal{B}(K)$ by the surjectivity of $\Phi$. This implies that $\Phi(I)=c I$ for some $c \in \mathbb{C}$. Furthermore, $c \neq 0$. Indeed, if $c=0$, then $\Phi(I)=0$. Thus by (3.3) we have $\Phi(R)^{2}=0$ for all idempotents $R$, which implies that $\Phi(C)^{2}=0$ for all self-adjoint operators $C$ by the boundedness of $\Phi$. Hence $\Phi(T)^{2}=0$ for all $T \in \mathcal{B}(H)$, which contradicts the surjectivity of $\Phi$.

Therefore, with no loss of generality, we may assume $\Phi(I)=I$. Thus, by (3.3) again, $\Phi$ is idempotent preserving. Let $A \in \mathcal{B}(H)$ be self-adjoint and $A=\sum_{i=1}^{n} t_{i} P_{i}$ where $t_{i} \in \mathbb{R}$ and $P_{i}$ are pairwise orthogonal projections. Since $\Phi$ maps mutually orthogonal projections to mutually orthogonal idempotents, $\Phi\left(A^{2}\right)=\Phi(A)^{2}$. Now, because the set of self-adjoint elements that are finite real linear combinations of orthogonal projections is dense in the set of all self-adjoint elements in $\mathcal{B}(H)$, we see that $\Phi\left(A^{2}\right)=\Phi(A)^{2}$ for all self-adjoint $A$ in $\mathcal{B}(H)$ by the boundedness of $\Phi$. Replacing $A$ by $A+B$ where both $A$ and $B$ are self-adjoint, we get $\Phi(A B+B A)=\Phi(A) \Phi(B)+\Phi(B) \Phi(A)$. Since every $T \in \mathcal{B}(H)$ can be written in the form $T=A+i B$ with $A$ and $B$ self-adjoint, the last relations imply that $\Phi\left(T^{2}\right)=\Phi(T)^{2}$. So $\Phi$ is Jordan.

Since $\mathcal{B}(K)$ is a prime ring, by [9, Thm. 3.1], $\Phi$ is either a homomorphism or an anti-homomorphism. If $\Phi$ is not injective, then $\operatorname{ker} \Phi$ is a nonzero closed ideal in $\mathcal{B}(H)$. Since the smallest nontrivial closed ideal of $\mathcal{B}(H)$ is the ideal $\mathcal{K}(H)$ of compact operators, we have $\operatorname{ker} \Phi \supseteq \mathcal{K}(H)$. Hence $\Phi(T)=0$ for all compact operators $T \in \mathcal{B}(H)$. If $\Phi$ is injective, then $\Phi$ is either an isomorphism or an anti-isomorphism. Since every isomorphism or anti-isomorphism from $\mathcal{B}(H)$ onto $\mathcal{B}(K)$ is spatial, there exists an invertible operator $A \in \mathcal{B}(H, K)$ such that $\Phi(T)=A T A^{-1}$ for all $T \in \mathcal{B}(H)$ or $\Phi(T)=A T^{\operatorname{tr}} A^{-1}$ for all $T \in \mathcal{B}(H)$, where $T^{\mathrm{tr}}$ denotes the transpose of $T$ relative to an orthonormal basis in $H$.

REMARK 3.4. Claim 2 in the proof of Theorem 3.3 also answers affirmatively a question due to Šemrl [15], who showed that a unital linear bijection on $\mathcal{B}(H)$ is square-zero preserving if and only if it is either an automorphism or an anti-automorphism and he asked whether or not the unital assumption may be omitted. So we find that a linear bijection on 
$\mathcal{B}(H)$ is square-zero preserving if and only if it is either an automorphism or an anti-automorphism multiplied by a nonzero scalar.

Proof of Theorem 3.1. $(2) \Rightarrow(3) \Rightarrow(4) \Rightarrow(5) \Rightarrow(1)$ are obvious. $(1) \Rightarrow(2)$ follows from Theorem 3.3.

Note that if the Hilbert space $H$ is separable, then $\Phi$ is injective in Theorem 3.3. In fact, if $\Phi$ is not injective, then $\mathcal{B}(H)$ is isomorphic to the quotient algebra $\mathcal{B}(H) / \mathcal{K}(H)$, which contradicts the fact that $\mathcal{B}(H) / \mathcal{K}(H)$ is simple. So we have the following corollary which generalizes $[14$, Thm. 2$]$ by omitting the unital assumption.

COROLlary 3.5. Let $H$ and $K$ be two infinite-dimensional Hilbert spaces with $H$ separable. Assume that $\Phi: \mathcal{B}(H) \rightarrow \mathcal{B}(K)$ is a surjective linear map. Then $\Phi$ is spectrally bounded if and only if there exists a nonzero complex number $d$ and an invertible operator $A \in \mathcal{B}(H, K)$ such that either $\Phi(T)=$ $d A T A^{-1}$ for all $T \in \mathcal{B}(H)$ or $\Phi(T)=d A T^{\mathrm{tr}} A^{-1}$ for all $T \in \mathcal{B}(H)$, where $T^{\mathrm{tr}}$ denotes the transpose of $T$ relative to a fixed but arbitrary orthonormal basis of $H$.

REMARK 3.6. Our proofs still work if $\mathcal{B}(K)$ is replaced by a standard operator algebra acting on a complex Banach space. So the results in this section also hold true when $\Phi$ is a linear map from $\mathcal{B}(H)$ onto a standard operator algebra $\mathcal{B}$.

Acknowledgments. We would like to thank the referee who pointed out Šemrl's paper [14] to us.

\section{References}

[1] B. Aupetit, Spectrum-preserving linear mappings between Banach algebras or Jordan-Banach algebras, J. London Math. Soc. (2) 62 (2000), 917-924.

[2] B. Aupetit, A Primer on Spectral Theory, Springer, New York, 1991.

[3] B. Aupetit and H. du T. Mouton, Spectrum preserving linear mappings in Banach algebras, Studia Math. 109 (1994), 91-100.

[4] M. Brešar and P. Šemrl, Invertibility preserving maps preserve idempotents, Michigan Math. J. 45 (1998), 483-488.

[5] -, - , Linear maps preserving the spectral radius, J. Funct. Anal. 142 (1996), 360368.

[6] M.-D. Choi, D. Hadwin, E. Nordgren, H. Radjavi and P. Rosenthal, On positive linear maps preserving invertibility, J. Funct. Anal. 59 (1984), 462-469.

[7] J. L. Cui and J. C. Hou, A characterization of homomorphisms between Banach algebras, Acta Math. Sinica, to appear.

[8] - - - Linear maps between Banach algebras compressing certain spectral functions, preprint.

[9] I. N. Herstein, Topics in Ring Theory, Springer, Berlin, 1991.

[10] J. C. Hou, Spectrum-preserving elementary operators on $\mathcal{B}(X)$, Chinese Ann. Math. Ser. B 19 (1998), 511-516. 
[11] A. A. Jafarian and A. R. Sourour, Spectrum-preserving linear maps, J. Funct. Anal. 66 (1986), 255-261.

[12] C. Pearcy and D. Topping, Sums of small numbers of idempotents, Michigan Math. J., 14 (1967), 453-465.

[13] P. Šemrl, Two characterizations of automorphisms on $\mathcal{B}(X)$, Studia Math. 105 (1993), 143-149.

[14] - Spectrally bounded linear maps on $\mathcal{B}(H)$, Quart. J. Math. Oxford 49 (1998), $87-92$.

[15] —, Linear mappings that preserve operators annihilated by a polynomial, J. Operator Theory 36 (1996), 45-58.

[16] A. R. Sourour, Invertibility preserving linear maps on $L(X)$, Trans. Amer. Math. Soc. 348 (1996), 13-30.

[17] Q. Wang and J. C. Hou, Point-spectrum-preserving elementary operators on $\mathcal{B}(H)$, Proc. Amer. Math. Soc. 126 (1998), 2083-2088.

[18] X. L. Zhang and J. C. Hou, Positive elementary operators compressing spectrum, Chinese Sci. Bull. 42 (1997), 270-274.

Jianlian Cui

Institute of Mathematics

Chinese Academy of Sciences

Beijing 100080, P.R. China

Current address:

Department of Applied Mathematics

Taiyuan University of Technology

Taiyuan 030024, P.R. China

Department of Mathematics

Shanxi Teachers University

Linfen 041004, P.R. China

E-mail: cuijl@dns.sxtu.edu.cn
Jinchuan Hou

Department of Mathematics Shanxi Teachers University Linfen 041004, P.R. China

Current address:

Department of Mathematics

Shanxi University Taiyuan 030000, P. R. China

E-mail: jhou@dns.sxtu.edu.cn

Received February 12, 2001

Revised version August 20, 2001 